\title{
The Role of A-Kinase Anchoring Proteins in cAMP-Mediated Signal Transduction Pathways
}

\author{
Neal M. Alto and John D. Scott* \\ Howard Hughes Medical Institute, Vollum Institute, \\ Oregon Health and Science University, Portland, OR
}

\begin{abstract}
Compartmentalization of signal transduction enzymes is an important mechanism of cellular signaling specificity. This occurs through the interaction of enzymes with scaffolding or anchoring proteins. To date, one of the best-studied examples of kinase anchoring is the targeting of protein kinase A to cellular locations through its association with A-kinase anchoring proteins (AKAPs). AKAPs mediate a high-affinity interaction with the type II regulatory subunit of protein kinase A for the purpose of localizing the kinase to pools of cyclic adenosine monophosphate and within proximity of preferred substrates. Furthermore, AKAPs can organize entire signaling complexes made up of kinases, phosphatases, signaling enzymes, and additional regulatory proteins.
\end{abstract}

Index Entries: cAMP-dependent protein kinase (PKA); A-kinase anchoring protein; kinase; phosphatase; cAMP.

\section{INTRODUCTION}

Extracellular signals, such as hormones, neurotransmitters, and growth factors, regulate a wide variety of cellular activities, including ion channel modulation, neuronal excitation, cell growth, and cell differentiation (1). Intracellular transduction systems receive these signals via receptors and transmit them quickly and precisely, resulting in the amplification of specific biological responses. Cells often are exposed to

\footnotetext{
*Author to whom all correspondence and reprint requests should be addressed. E-mail address: scott@ ohsu.edu
}

several messengers simultaneously; therefore, maintaining the fidelity of these networks is crucial in eliciting the appropriate physiological response. Doing so requires the accurate selection of effector molecules for activation and deactivation, often by phosphorylation and dephosphorylation events. A principal strategy in achieving this selection of specificity is compartmentalization of signaling enzymes (2-4). This review focuses on introducing the conceptual advances that have resulted from studying the localization of the cyclic adenosine monophosphate (cAMP)dependent protein kinase A (PKA) mediated by A-kinase anchoring proteins (AKAPs). 


\section{CYCLIC AMP-DEPENDENT PROTEIN KINASE}

The role of cAMP as a second messenger was first discovered in 1957 (5). Since that time, more than 78,000 papers have been published on this subject. From these studies, many of the molecular mechanisms and physiological processes governed by cAMP have been determined. For example, cAMP is generated after hormonal activation of $G$ protein-coupled receptors (GPCRs) (6). Hormone binding to a receptor leads to conformational change, followed by the conversion of the heterotrimeric $\mathrm{G}$ protein subunit, Gas, into its active guanosine 5'-monophosphate-bound state (7). Activated Gos is released from the $\beta \gamma$ subunits and activates the enzyme adenylyl cyclase, which converts adenosine triphosphate to the second messenger cAMP. The primary downstream target of CAMP is the cAMP-dependent PKA (8). However, it should be noted that cAMP has roles independent of PKA, including the regulation CAMP-activated ion channels and a recently discovered family of Ras family-guanine nucleotide exchange factors (9-11). The use of pharmacological inhibitors has aided in distinguishing between the physiological processes regulated by PKA vs those of other cAMP-binding proteins (12).

PKA is a serine/threonine kinase composed of two catalytic (C) subunits that are held in an inactive state by association with a regulatory (R) subunit dimer (13-16). The catalytic subunits (C) are expressed from three different genes: $C \alpha, C \beta$, and $C \gamma$, whereas the regulatory subunits $(\mathrm{R})$ are expressed from four different genes: RI $\alpha$, RI $\beta$, RII $\alpha$, and RII $\beta$ (17-19). The R subunit is a modular protein containing an $\mathrm{NH}_{2}$-terminal homodimerization domain, pseudosubstrate site that serves as a principal contact site for the $C$ subunit, and two cAMP binding sites. PKA is activated by the binding of cAMP to the R subunits $(20,21)$, which relieves the autoinhibitory contact, allowing the $C$ subunits to dissociate and phosphorylate local substrates $(22,23)$. Two forms of the heterotetrameric PKA holoenzyme exist: type I
(RI $\alpha$ and RI $\beta$ dimer) and type II (RII $\alpha$ and RII $\beta$ dimer). Type I PKA is predominantly cytoplasmic, whereas type II PKA associates with specific cellular structures and organelles (24).

One of the most fascinating and complex features of PKA signaling is that it can regulate a multitude of physiological processes. For example, in many neuronal cell types, PKA can regulate both gene expression in the nucleus and synaptic transmission by modifying the excitation state of ion channels at the synapse. This occurs, in part, because PKA has broad substrate specificity. In the example shown previously, PKA alters gene expression by directly phosphorylating the transcription factor CREB in the nucleus (25), and at dendrites PKA can phosphorylate and regulate the activation state of AMPA-type glutamate receptors (26). In fact, approximately 150 physiologically relevant PKA substrates have been identified (27); however, the mechanism by which PKA distinguishes between substrates to elicit a given biological response continues to be the subject of intense investigation. There are two prevailing theories to explain how this is accomplished. First, it is possible that the cell can create localized gradients of cAMP that only activate a subset of PKA molecules (6). This has been demonstrated experimentally and may require the selective and localized activation of adenylyl cyclases and phosphodiesterases, the enzymes that catalyze cAMP metabolism. One tenet of this hypothesis is that PKA must be compartmentalized, creating pools of active enzyme that are spatially restricted within the cell (28). The second prevailing theory is that PKA can be physically coupled to its substrate, thus decreasing the likelihood of spurious phosphorylation events (2). Both of these theories are probably correct, and exciting new evidence suggests that these two types of regulation are not mutually exclusive. These topics will be discussed in the following sections.

\section{A-KINASE ANCHORING PROTEINS}

Association with AKAPs leads to discrete localization of PKA within the cell. The first 
AKAPs were discovered as contaminants of type II PKA holoenzyme preparations (29-31) and the family has since grown to include more than 20 genes. With only one known exception, AKAPs contain an amphipathic helix that functions to interact with the binding surface formed by the amino termini of the PKA-RII dimer (32-34). Solution structure analysis of the RII binding domain from two different AKAPs indicates that this interaction occurs through hydrophobic contacts (35). Although AKAPs share this functionally similar 14 to 18 amino acid domain, they are structurally diverse proteins that are found in various cell types and subcellular locations. All AKAPs contain targeting domains that localize to different organelles and subcellular structures (2), thus providing a mechanism to control the intracellular localization of PKA. Furthermore, it is now clear that AKAPs can coordinate the assembly of signaling complexes by simultaneously tethering enzymes that can directly or indirectly regulate the phosphorylation state of a variety of substrates (2).

\section{PKA ANCHORING AND CELLULAR PHYSIOLOGY}

Insights into the spatial and temporal regulation of PKA has spurred the acceptance of the "anchoring hypothesis" to explain the multiple roles of this kinase in physiology. One tenet of the anchoring hypothesis is that PKA signaling specificity is achieved through AKAP-mediated compartmentalization of the kinase. An early demonstration the importance of PKA anchoring came from studies of the AMPAtype glutamate receptor in hippocampal neurons. PKA can directly phosphorylate the AMPA channel specifically at serine 845 , leading to a potentiation of the channel $(36,37)$. Rosenmund et al. demonstrated that this type of regulation requires AKAP-mediated targeting of PKA to the AMPA channel (38). They found that disrupting anchored PKA with a peptide that competitively displaces the kinase from AKAPs leads to dephosphorylation of serine 845 and subsequent time-dependent decrease in peak current amplitude. Since this initial study, other investigators have performed experiments to suggest that AKAP79 is the endogenous AKAP that is responsible for anchoring PKA and the phosphatase PP2B to the AMPA-receptor $(39,40)$. These studies have implicated AKAP79 in such processes as longterm depression (40), a state of synaptic plasticity that serves as a model for studying the formation and storage of memories in the human brain.

Since the initial use of Ht31, a member of the AKAP family, as an inhibitor of PKA-AKAP interactions in vivo, analogous experiments have been performed to implicate AKAPs in numerous physiological processes. For example, PKA anchoring is required for normal contractility of cardiac myocytes on stimulation of the $\beta$-adrenergic receptor (41). In addition, PKA anchoring is necessary for the cell surface expression of the water channel, aquaporin-2, in renal collecting duct principal cells (42). Finally, the roles for AKAPs in animal behavioral models are now being tested. Moita et al. infused Ht31 into cells within the amygdala of adult rats before a fear-conditioning protocol. They found AKAP-mediated anchoring in the lateral amygdala is necessary for the consolidation, but not acquisition, of conditioned fear (43). Together, these examples highlight the importance of using AKAP-derived reagents to further define the mechanisms that regulate cAMP signaling events in cellular and animal models.

\section{AKAPS LOCALIZE PKA TO CAMP GRADIENTS}

Although it is clear that AKAP-mediated PKA anchoring is a physiologically important process, the consequences of anchoring at the molecular level are only now beginning to be appreciated. For example, AKAPs can directly link PKA to upstream or downstream regulatory proteins $(44,45)$. A connection between PKA and the cAMP-degrading enzyme, phosphodiesterase, can be made by various AKAPs 
$(44,46)$. This could ensure that the kinase is transiently exposed to cAMP gradients and may provide a mechanism to favor the efficient catalytic activation of the kinase. Interestingly, this type of regulation has been elegantly described for two different AKAPs and in a unique study using chimeric proteins. Dodge et al. found that the muscle-selective mAKAP directly binds PKA and a splice variant of the cAMP-specific, type 4 phosphodiesterase, PDE4D3 (44). Subsequently, Tasken et al. reported the interaction of PDE4D3 with AKAP450, a large centrosomal AKAP found in Sertoli cells (46). Both studies suggest that the role of PDE4D3 within these complexes is to depress cAMP levels within the vicinity of anchored PKA. At rest, PDE4D3 inhibits basal PKA activity associated with $\mathrm{mAKAP}$, possibly acting to dampen noise and increase gain in the system. Furthermore, PKA phosphorylation is known to upregulate PDE4D3 activity twofold to threefold, establishing a negative feedback loop that rapidly terminates the CAMP signal.

Recently, Zaccolo and Pozzan demonstrated that pools of CAMP generated through a specific GPCR could selectively activate anchored PKA (28). This group developed a chimeric cAMP reporter system by which the catalytic subunit of PKA is fused to yellow fluorescent protein (YFP) and the type II regulatory subunit is fused to cyan fluorescent protein (CFP). When in close proximity, these chimeric proteins undergo fluorescent resonance energy transfer (FRET). FRET occurs on excitation of the donor CFP with $440 \mathrm{~nm}$ light, and part of the excitation energy is transferred to the acceptor YFP, which then emits $545 \mathrm{~nm}$ light. Detection of the excited YFP indicates that the donor and acceptor molecules are within $50 \mathrm{~nm}$ of each other, and therefore, in the case of PKA, the $C$ subunit and $R$ subunit form the inactive heterotetramer. Expression of the cAMP reporter chimera in cardiac myocytes, a cell type that is highly regulated by cAMP, resulted in FRET detection throughout the T-tubule system. This indicates that the chimeric proteins are anchored to the Ttubules and that they form the heterotetrameric holoenzyme complex. On activation of the $\mathrm{Go}_{\mathrm{s}}{ }^{-}$ coupled $\beta$-adrenergic receptor ( $\beta$-AR), a loss in FRET signal and thus PKA activation, was observed. Upon deletion of the AKAP binding domain of the RII-CFP chimera, a PKA molecule was created that could not target to the Ttubules via AKAPs. No reduction in FRET signal was detected on $\beta$-AR stimulation under these conditions. Together, these data suggest that $\beta$-AR stimulation in cardiac myocytes leads to a compartmentalized increase of cAMP in the vicinity of the T-tubules, thus allowing localized activation of PKA.

\section{AKAPS DIRECTLY COUPLE SIGNAL TRANSDUCTION COMPLEXES TO DOWNSTREAM EFFECTORS}

A newly recognized duty for AKAPs is to coordinate signaling complexes by recruiting multiple signaling enzymes near potential substrates. This effectively bridges the gap between upstream activators and downstream targets. AKAP79, AKAP220, and yotiao have already been shown to function in this capacity $(39,45,47,48)$. As discussed previously, AKAP79 links PKA directly to the AMPA-type glutamate receptor. Recently it was demonstrated that AKAP220 interacts with GSK-3beta and promotes its phosphorylation by PKA (47). Finally, in neurons, yotiao targets both PKA and the phosphatase PP1 to the $\mathrm{C} 1$ exon containing NR1 subunit of the N-methyl-D-aspartate (NMDA) receptor $(48,49)$. The localized, active PP1 maintains the NMDA receptor in a depressed state, and on cAMP elevation and subsequent PKA activation, the NMDA receptor current is increased by $55 \%$. These examples highlight a growing theme in signal transduction: multiple kinases, phosphatases, and signal transduction enzymes can be directly tethered to downstream targets.

Directly tethering kinases to their substrates may impart both spatial and temporal specificity to a signal transduction system. This idea was tested using a variation of the FRET technology described previously. Instead of developing a cAMP reporter, Zhang et al. designed a 
PKA phosphorylation indicator (50). They built a chimeric protein consisting of a CFP molecule followed by the phospho-amino acid binding region of 14-3-3, a PKA substrate peptide and a YFP molecule. PKA phosphorylation of the substrate peptide results in an intramolecular interaction between the 14-3-3 module and the phospho-amino acid. This brings the CFP and YFP moieties close enough to detect a FRET signal. In vivo, direct activation of PKA leads to a rapid and reversible increase in FRET. Interestingly, if PKA is targeted to this chimera by introducing an RII-binding domain from a known AKAP, the time course for phosphorylation of the reporter is significantly shortened. This occurs as a direct result of coupling PKA to the substrate within the chimera. Together, these results suggest that AKAPs may not only allow spatial restriction of the enzyme, but also may impart temporal control of the system.

\section{CONCLUSION}

AKAP-mediated mechanisms of action are beginning to emerge from the many studies over the past decade. It is now clear that AKAPs can localize PKA in close proximity to physiological substrates and to areas where PKA can be modulated by localized cAMP pools. Furthermore, recruitment of phosphatases is critical for the bidirectional phosphotransfer reactions on substrates. It is also clear that AKAPs can interact with enzymes that are not necessarily linked to cAMP signaling. This suggests that AKAPs provide a platform to integrate multiple signal transduction inputs to coordinate a physiological response. Many challenges still remain for the AKAP field. First, it is imperative that all AKAPs be identified. This is a difficult problem because there no methods for searching computer databases for novel AKAPs. However, with more advanced bioinformatic software and the ability to screen entire genomes, these approaches may yet be possible. Second, it will be important to distinguish PKA signaling events that are anchorage-dependent versus those that are not. This will include the identification of PKA anchoring-dependent substrates and determining the physiological processes mediated by anchored PKA. Third, identifying the full complement of AKAP binding proteins will be indispensable for elucidation of signal transduction pathways mediated by AKAPs. Gene knockout and specific inhibitory reagents will be useful for these types of experiments.

\section{REFERENCES}

1. Sutherland, E. W. (1972) Studies on the mechanism of hormone action. Science 171, 401-408.

2. Colledge, M. and Scott, J. D. (1999) AKAPs: from structure to function. Trends Cell Biol. 9, 216-221.

3. Diviani, D. and Scott, J. D. (2001) AKAP signaling complexes at the cytoskeleton. J. Cell. Sci. 114, 1431-1437.

4. Pawson, T. and Scott, J. D. (1997) Signaling through scaffold, anchoring, and adaptor proteins. Science 278, 2075-2080.

5. Sutherland, E. W. and Rall, T. W. (1957) The properties of an adenine ribonucleotide produced with cellular particles, ATP, $\mathrm{Mg}^{++}$, and epinephrine or glucagon. J. Amer. Chem. Soc. 79, 3608-3610.

6. Beavo, J. A. and Brunton, L. L. (2002) Cyclic nucleotide research—still expanding after half a century. Nat. Rev. Mol. Cell Biol. 3, 710-718.

7. Pierce, K. L., Premont, R. T., and Lefkowitz, R. J. (2002) Seven-transmembrane receptors. Nat. Rev. Mol. Cell Biol. 3, 639-650.

8. Walsh, D. A., Perkins, J. P., and Krebs, E. G. (1968) An adenosine 3',5'-monophosphatedependent protein kinase from rabbit skeletal muscle. J. Biol. Chem. 243, 3763-3765.

9. de Rooij, J., Zwartkruis, F. J., Verheijen, M. H., Cool, R. H., Nijman, S. M., Wittinghofer, A., et al. (1998) Epac is a Rap1 guanine-nucleotideexchange factor directly activated by cyclic AMP. Nature 396, 474-477.

10. Kaupp, U. B. and Seifert, R. (2002) Cyclic nucleotide-gated ion channels. Physiol. Rev. 82, 769-824.

11. Kawasaki, H., Springett, G. M., Mochizuki, N., Toki, S., Nakaya, M., Matsuda, M., et al. (1998) A family of cAMP-binding proteins that directly activate Rap1. Science 282, 2275-2279. 
12. Smith, M. K., Colbran, R. J., and Soderling, T. R. (1990) Specificities of autoinhibitory domain peptides for four protein kinases. Implications for intact cell studies of protein kinase function. J. Biol. Chem. 265, 1837-1840.

13. Corbin, J. D. and Keely, S. L. (1977) Characterization and regulation of heart adenosine 3': 5'- monophosphate-dependent protein kinase isozymes. J. Biol. Chem. 252, 910-918.

14. Corbin, J. D., Soderling, T. R., and Park, C. R. (1973) Regulation of adenosine 3',5'-monophosphate-dependent protein kinase. J. Biol. Chem. 248, 1813-1821.

15. Potter, R. L., Stafford, P. H., and Taylor, S. (1978) Regulatory subunit of cyclic AMP-dependent protein kinase I from porcine skeletal muscle: purification and proteolysis. Arch. Biochem. Biophys. 190, 174-180.

16. Potter, R. L. and Taylor, S. S. (1979) Relationships between structural domains and function in the regulatory subunit of cAMPdependent protein kinases I and II from porcine skeletal muscle. J. Biol. Chem. 254, 2413-2418.

17. Chrivia, J. C., Uhler, M. D., and McKnight, G. S. (1988) Characterization of genomic clones coding for the $\mathrm{Ca}$ and $\mathrm{Cb}$ subunits of mouse cAMPdependent protein kinase. J. Biol. Chem. 263, 5739-5744.

18. Lee, D. C., Carmichael, D. F., Krebs, E. G., and McKnight, G. S. (1983) Isolation of cDNA clone for the type I regulatory subunit of bovine cAMP-dependent protein kinase. Proc. Natl. Acad. Sci. U S A 80, 3608-3612.

19. Scott, J. D., Zoller, M. J., Glaccum, M. B., Uhler, M. D., Helfman, D. M., McKnight, G. S., et al. (1987) The molecular cloning of a type II regulatory subunit of the cAMP-dependent protein kinase from rat skeletal muscle and mouse brain. Proc. Natl. Acad. Sci. U S A 84, 5192-5196.

20. Su, Y., Dostmann, W. R. G., Herberg, F. W., Durick, K., Xuong, N. H., Ten Eyck, L., et al. (1995) Regulatory subunit of protein kinase A: structure of deletion mutant with cAMP binding proteins. Science 269, 807-813.

21. Su, Y., Taylor, S. S., Dostmann, W. R. G., Xuong, N. H., and Varughese, K. I. (1993) Crystallization of a deletion mutant of the Rsubunit of cAMP-dependent protein kinase. J. Mol. Biol. 230, 1091-1093.

22. Gibbs, C. S., Knighton, D. R., Sowadski, J. M., Taylor, S. S., and Zoller, M. J. (1992) Systematic mutational analysis of cAMP-dependent protein kinase identifies unregulated catalytic sub- units and defines regions important for the recognition of the regulatory subunit. J. Biol. Chem. 267, 4806-4814.

23. Wang, Y., Scott, J. D., McKnight, G. S., and Krebs, E. G. (1991) A constitutively active holoenzyme from the cAMP-dependent protein kinase. Proc. Natl. Acad. Sci. U S A 88, 2446-2450.

24. Scott, J. D. (1991) Cyclic nucleotide-dependent protein kinases. Pharmacol. Ther. 50, 123-145.

25. Gonzalez, G. A., Yamamoto, K. K., Fischer, W. H., Karr, D., Menzel, P., Biggs, W., et al. (1989) A cluster of phosphorylation sites on the cyclic AMP-regulated nuclear factor CREB predicted by its sequence. Nature 337, 749-752.

26. Banke, T.G., Bowie, D., Lee, H., Huganir, R. L., Schousboe, A., and Traynelis, S. F. (2000) Control of GluR1 AMPA receptor function by cAMP-dependent protein kinase. J. Neurosci. 20, 89-102.

27. Shabb, J. B. (2001) Physiological substrates of cAMP-dependent protein kinase. Chem. Rev. 101, 2381-2411.

28. Zaccolo, M. and T. Pozzan. (2002) Discrete microdomains with high concentration of cAMP in stimulated rat neonatal cardiac myocytes. Science 295, 1711-1715.

29. Rubin, C. S., Rangel-Aldao, R., Sarkar, D., Erlichman, J., and Fleischer, N. (1979) Characterization and comparison of membrane-associated and cytosolic cAMP-dependent protein kinases. J. Biol. Chem. 254, 3797-3805.

30. Theurkauf, W. E. and Vallee, R. B. (1982) Molecular characterization of the cAMP-dependent protein kinase bound to microtubule-associated protein 2. J. Biol. Chem. 257, 3284-3290.

31. Vallee, R. B., DiBartolomeis, M. J., and Theurkauf, W. E. (1981) A protein kinase bound to the projection portion of MAP 2 (microtubule-associated protein 2). J. Cell. Biol. 90, 568-576.

32. Carr, D. W., Stofko-Hahn, R. E., Fraser, I. D. C., Bishop, S. M., Acott, T. S., Brennan, R. G., et al. (1991) Interaction of the regulatory subunit (RII) of cAMP-dependent protein kinase with RIIanchoring proteins occurs through an amphipathic helix binding motif. J. Biol. Chem. 266, 14188-14192.

33. Diviani, D., Langeberg, L. K., Doxsey, S. J., and Scott, J. D. (2000) Pericentrin anchors protein kinase $\mathrm{A}$ at the centrosome through a newly identified RII-binding domain. Curr. Biol. 10, 417-420. 
34. Newlon, M. G., Roy, M., Hausken, Z. E., Scott, J. D., and Jennings, P. A. (1997) The A-kinase anchoring domain of Type IIa cAMP-dependent protein kinase is highly helical. J. Biol. Chem. 272, 23637-23644.

35. Newlon, M. G., Roy, M., Morikis, D., Carr, D. W., Westphal, R., Scott, J. D., et al. (2001) A novel mechanism of PKA anchoring revealed by solution structures of anchoring complexes. EMBO J. 20, 1651-1662.

36. Blackstone, C., Murphy, T. H., Moss, S. J., Baraban, J. M., and Huganir, R. L. (1994) Cyclic AMP and synaptic activity-dependent phosphorylation of AMPA- preferring glutamate receptors. J. Neurosci. 14, 7585-7593.

37. Roche, K. W., O'Brien, R. J., Mammen, A. L., Bernhardt, J., and Huganir, R. L. (1996) Characterization of multiple phosphorylation sites on the AMPA receptor GluR1 subunit. Neuron 16, 1179-1188.

38. Rosenmund, C., Carr, D. W., Bergeson, S. E., Nilaver, G., Scott, J. D., and Westbrook, G. L. (1994) Anchoring of protein kinase A is required for modulation of AMPA/kainate receptors on hippocampal neurons. Nature 368, 853-856.

39. Colledge, M., Dean, R. A., Scott, G. K., Langeberg, L. K., Huganir, R. L., and Scott, J. D. (2000) Targeting of PKA to glutamate receptors through a MAGUK-AKAP complex . Neuron 27, 107-119.

40. Tavalin, S. J., Colledge, M., Hell, J. W., Langeberg, L.K., Huganir, R. L., and Scott, J. D. (2002) Regulation of GluR1 by the A-kinase anchoring protein 79 (AKAP79) signaling complex shares properties with long-term depression. J. Neurosci. 22, 3044-3051.

41. Fink, M. A., Zakhary, D. R., Mackey, J. A., Desnoyer, R. W., Apperson-Hansen, C., Damron, D. S., et al. (2001) AKAP-mediated targeting of protein kinase A regulates contractility in cardiac myocytes. Circ. Res. 88, 291-297.

42. Klussmann, E., Maric, K., Wiesner, B., Beyermann, M., and Rosenthal, W. (1999) Protein kinase $\mathrm{A}$ anchoring proteins are required for vasopressin- mediated translocation of aquaporin-2 into cell membranes of renal principal cells. J. Biol. Chem. 274, 4934-4938.

43. Moita, M. A., Lamprecht, R., Nader, K., and LeDoux, J. E. (2002) A-kinase anchoring proteins in amygdala are involved in auditory fear memory. Nat. Neurosci. 5, 837-838.

44. Dodge, K. L., Khouangsathiene, S., Kapiloff, M. S., Mouton, R., Hill, E. V., Houslay, M. D., et al. (2001) mAKAP assembles a protein kinase A/PDE4 phosphodiesterase cAMP signaling module. EMBO J. 20, 1921-1930.

45. Fraser, I., Cong, M., Kim, J., Rollins, E., Daaka, Y., Lefkowitz, R., et al. (2000) Assembly of an AKAP/beta2-adrenergic receptor signaling complex facilitates receptor phosphorylation and signaling. Curr. Biol. 10, 409-412.

46. Tasken, K. A., Collas, P., Kemmner, W. A., Witczak, O., Conti, M., and Tasken, K. (2001) Phosphodiesterase $4 \mathrm{D}$ and protein kinase a type II constitute a signaling unit in the centrosomal area. J. Biol. Chem. 276, 21999-22002.

47. Tanji, C., Yamamoto, H., Yorioka, N., Kohno, N., Kikuchi, K., and Kikuchi, A. (2002) A-kinase anchoring protein AKAP220 binds to glycogen synthase kinase- 3beta (GSK-3beta) and mediates protein kinase A-dependent inhibition of GSK-3beta. J. Biol. Chem. 277, 36955-36961.

48. Westphal, R. S., Tavalin, S. J., Lin, J. W., Alto, N. M., Fraser, I. D., Langeberg, L. K., et al. (1999) Regulation of NMDA receptors by an associated phosphatase-kinase signaling complex. Science 285, 93-96.

49. Lin, J. W., Wyszynski, M., Madhavan, R., Sealock, R., Kim, J. U., and Sheng, M. (1998) Yotiao, a novel protein of neuromuscular junction and brain that interacts with specific splice variants of NMDA receptor subunit NR1. J. Neurosci. 18, 2017-2027.

50. Zhang, J., Ma, Y., Taylor, S. S., and Tsien, R. Y. (2001) Genetically encoded reporters of protein kinase A activity reveal impact of substrate tethering. Proc. Natl. Acad. Sci. U S A 98, 14997-15002. 